\title{
Review
}

Ana Lisica ${ }^{a}$ and Stephan W. Grill*

\section{Optical tweezers studies of transcription by eukaryotic RNA polymerases}

DOI 10.1515/bmc-2016-0028

Received December 1, 2016; accepted January 10, 2017

Abstract: Transcription is the first step in the expression of genetic information and it is carried out by large macromolecular enzymes called RNA polymerases. Transcription has been studied for many years and with a myriad of experimental techniques, ranging from bulk studies to high-resolution transcript sequencing. In this review, we emphasise the advantages of using single-molecule techniques, particularly optical tweezers, to study transcription dynamics. We give an overview of the latest results in the single-molecule transcription field, focusing on transcription by eukaryotic RNA polymerases. Finally, we evaluate recent quantitative models that describe the biophysics of RNA polymerase translocation and backtracking dynamics.

Keywords: optical tweezers; RNA polymerases; singlemolecule techniques; transcription.

\section{Introduction}

Transcription of the genetic information from DNA into RNA is the first step of gene expression and a central point of cellular regulation. Transcription is performed by macromolecular enzymes called RNA polymerases (RNAPs) that move stepwise along the DNA template and produce a complementary RNA molecule. The process is traditionally divided into three main phases: initiation, in

aPresent address: London Centre for Nanotechnology, University College London, London WC1H OAH, UK

*Corresponding author: Stephan W. Grill, BIOTEC, Technical University Dresden, Tatzberg 47/49, D-01307 Dresden, Germany; and Max Planck Institute of Molecular Cell Biology and Genetics, Pfotenhauerstraße 108, D-01307 Dresden, Germany, e-mail: stephan.grill@tu-dresden.de

Ana Lisica: BIOTEC, Technical University Dresden, Tatzberg 47/49, D-01307 Dresden, Germany; and Max Planck Institute of Molecular Cell Biology and Genetics, Pfotenhauerstraße 108, D-01307 Dresden, Germany which the polymerase binds to a specific sequence in the DNA and starts the synthesis of RNA; elongation, during which the polymerase moves on the DNA template and produces a complementary RNA sequence; and termination, in which the polymerase dissociates from the DNA and releases an RNA transcript. All phases of transcription are highly-regulated processes with specific transcription factors acting together with the enzyme to make transcription a highly efficient process.

Gene transcription has been investigated with a number of different techniques. Classic biochemical assays gave insight into elongation dynamics of RNAPs and have identified proteins involved in transcription; structural studies gave detailed, albeit static, intermediate structures of RNAPs and accompanying transcription factors [reviewed in Ref. (1)]; and high-resolution genomic approaches and imaging techniques provided the kinetics of the polymerase movement and the dynamics of cotranscriptional processes, such as splicing and chromatin remodelling [reviewed in Refs. $(2,3)]$.

With the development of single-molecule biophysics techniques, it has become possible to observe and manipulate individual polymerases with very high spatial and temporal resolution. There are several advantages of using single-molecule techniques to study transcription dynamics. First, these techniques provide the ability to simplify the complex in vivo transcription machinery and to track and manipulate individual RNA polymerase molecules, one at a time, without the myriad of additional interactions that take place inside a cell $(4,5)$. Second, they enable access to the dynamics of single molecules, and thus allow us to circumvent the necessity to average over thousands of molecules in bulk experiments (4-6). Third, with single-molecule techniques it is possible to measure highly dynamic and fast movements, given the high spatiotemporal resolution that has become available (7). Fourth, individual molecules can be perturbed and manipulated in specific ways by force, and the response of an individual molecule to applied external force can be recorded (8-12). Therefore, single-molecule techniques enable the acquisition of precise and quantitative data, which allows the verification of biophysical theoretical models of transcription 
dynamics and regulation. This is needed for unravelling the complete spectrum of micromechanical events that underlie this fundamental biological process.

In this review, we focus on single-molecule optical tweezers studies of transcription [for a detailed review of other single-molecule techniques in transcription studies, please refer to Ref. (5)]. We give a brief summary of the methodology, give an overview of the past studies of transcription by bacterial and eukaryotic RNA polymerases and focus on recent advances in the field. The review is structured according to the phases of the transcription process: initiation, elongation and termination. We conclude by presenting current challenges of the optical tweezers technique, discuss future directions and consider potential integration with other techniques.

\section{Basics of optical tweezers}

Recent advances in physics and biology have led to the development of different single-molecule micromanipulation and detection techniques, such as single-molecule FRET measurements, optical and magnetic tweezers, atomic force microscopy, single-molecule fluorescent RNA detection methods. In this review, we focus on optical tweezers.

Optical tweezers are an instrument that uses highlyfocused laser light to trap micron-sized particles. The basic principle behind optical trapping is momentum conservation, and this can be illustrated best in the rayoptics regime. Light is composed of photons that are retarded in media that are optically more dense. If one takes a small dielectric particle with an increased index of refraction as compared to the surrounding medium, photons are refracted as they enter and exit the particle due to the retardation of photons in the optically denser medium. This decrease in light velocity causes a change in the direction at the interface, and thus a change of the momentum of photons. Due to momentum conservation, however, the dielectric particle must experience an equal and opposite change of momentum. In a highly focused laser beam of sufficient power, this can give rise to an attractive tracking force that will capture the dielectric particle in three-dimensional (3D) space. More precisely, gradient forces act to restore the position of the particle towards the centre of the focused laser beam. The photons are not only refracted but also reflected from the surface of the particle, giving rise to scattering forces that push the particle in the direction of propagation of light $(13,14)$. It is due to scattering forces that the dielectric particle is trapped slightly downstream of the centre of the beam.
To achieve stable trapping in three dimensions, a single laser beam is typically focused through a high numerical aperture lens. For small distances around the centre, the trap behaves like a linear Hooke's spring. The generated forces are proportional to the displacement of the object, according to $F=k x$, where $x$ denotes the displacement of the particle from the trap centre, $F$ is the optical force, and $k$ is an effective stiffness of the trap. Knowing the trap stiffness is, therefore, crucial for accurately determining the forces involved and is usually determined from thermal fluctuations of the position of the trapped particle [i.e. Brownian motion calibration; Refs. (15-17)]. Forces typically exerted by optical tweezers are on the piconewton level $(\mathrm{pN})$ and distances measured are on the nanometre level $(\mathrm{nm})$. These length- and forcescales make optical tweezers particularly useful for investigating the functions of cytoplasmic and DNA molecular motors, such as RNA polymerases.

In biological applications, the forces are usually not applied directly on the macromolecules of interest, but on glass or polystyrene micron-sized beads that are linked to them. Different experimental geometries were used in optical tweezers transcription experiments, e.g. single optical traps with macromolecules tethered directly to the surface of the chamber $(18,19)$ or systems where one particle is trapped in an optical trap and another one is held with a micropipette (20). Finally, the dual-trap experimental system uses a single trapping laser that is split by polarisation into two beams, each of which forms an optical trap. This configuration reduces the noise in the system, as beads are not attached to any surface, but are trapped free in solution. The data is recorded in each trap independently, thus only the anti-correlated data represents the signal and the correlated data can be attributed to noise (6).

In a typical dual-trap optical tweezers transcription experiment, the DNA tether is formed between the tagged polymerase attached to one bead (usually via a biotinstreptavidin interaction) and the tagged end of the DNA attached to another bead (usually via a digoxigenin-antidigoxigenin interaction) (Figure 1A). Depending on where the tagged DNA is located with respect to the polymerase, i.e. upstream or downstream of the polymerase, the forces applied on the enzyme can either assist or oppose the transcription. In the assisting force mode, the tagged DNA is upstream of the polymerase, therefore an active transcription by the enzyme increases the distance between the beads and reduces the force. In the opposing force mode, the DNA is labelled downstream of the transcribing polymerase, which results in decrease of the distance between the beads and increase of the force. Both configurations are used in optical tweezers transcription experiments. 
A

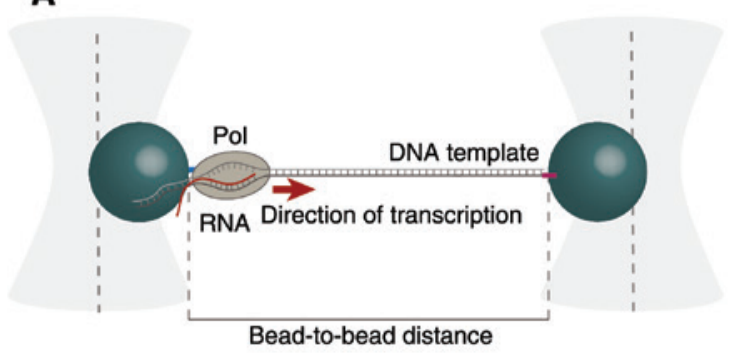

B

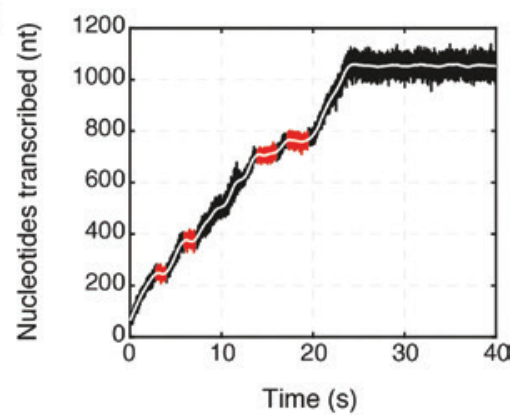

Figure 1: Dual-trap optical tweezers experimental set-up scheme and a representative RNA polymerase trajectory.

(A) Two optical traps (grey) are used to trap functionalised polystyrene beads, typically around $2 \mu \mathrm{m}$ in diameter (green). Tagged polymerase is attached to one bead and the labelled DNA is attached to another bead. In the opposing force mode experimental set-up shown here, the polymerase transcribes the DNA in between the two beads, therefore shortening the bead-to-bead distance during the experiment, while the forces applied to the enzyme are increased. Note: the scheme is not to scale. (B) Example optical tweezers transcription trace showing transcribed nucleotides over time of an individual transcribing RNA polymerase I. The raw unfiltered data is shown in black, with the filtered trace overlaid in white. Pauses are marked in red.

Opposing force mode is preferred for studying pausing and backtracking dynamics of RNA polymerases, as it was shown that the enzymes pause more with opposing forces $(11,21)$. Assisting force mode is often used in studies of transcription through nucleosomes, where the tension applied to the upstream DNA ensures there is no transfer of nucleosomes behind the polymerase (22-24).

\section{Studies of transcription initiation}

Transcription initiation is a highly regulated process during which an RNA polymerase binds to a promotor sequence of a gene and unwinds double-stranded DNA around the transcriptional start site to form a transcription bubble. Initiation in prokaryotes is assisted by a single transcription factor $-\sigma$ factor, while in eukaryotes it requires a myriad of proteins (25). Due to the complexity of the preinitiation complex (PIC) in eukaryotes, until recently, most of the single-molecule studies focused on prokaryotic transcription initiation. Studies using methods such as singlemolecule FRET and magnetic tweezers have showed that abortive initiation of bacterial RNA polymerase proceeds mainly through a scrunching mechanism, in which the downstream DNA is unwound and pulled into a stationary $\operatorname{RNAP}(26,27)$ and that most mature elongation complexes retain $\sigma 70$ factor throughout elongation (28). Furthermore, recent work revealed dynamics of the RNAP initiation complex (29) and the pausing of RNAP during initial transcription at lac promoters (30).

A recent study by Fazal and colleagues reported an extraordinary achievement of assembling a 32-protein pre-initiation complex (PIC), in real time, using optical tweezers to study the initiation of yeast RNA polymerase II (Pol II) (10). The complex consisted of Pol II and six general transcription factors, including TATA-binding protein, TFIIB, TFIIE, TFIIF, TFIIH and TFIIA and Sub1 (Figure 2A). The authors found that during initiation a large bubble is opened in the DNA, driven by a member of the PIC, the TFIIH helicase. Contrary to previous findings that Pol II produces only a short transcript during initiation, Fazal and colleagues found that Pol II synthesises $85 \mathrm{bp}$ on average, up to the length of the open bubble, followed by promotor escape (Figure 2B). These studies from yeast are likely to hold true even in metazoans, as it was shown in several species that the transcriptional start sites are located far from the TATA-boxes, therefore requiring a bigger bubble.

\section{Elongation phase}

After successful initiation, the RNA polymerase, the DNA and the nascent RNA form a stable transcription elongation complex (TEC) that moves downstream on the DNA, in the $5^{\prime}$ to $3^{\prime}$ direction, while incorporating complementary nucleotides in the growing RNA chain. A number of single-molecule studies provided great insights into the dynamics of transcription elongation, pausing, elongation in the presence of transcription factors and through nucleosomal templates.

\section{Elongation dynamics of RNA polymerases}

The first optical tweezers elongation studies were performed using bacterial RNA polymerase. Initial studies 

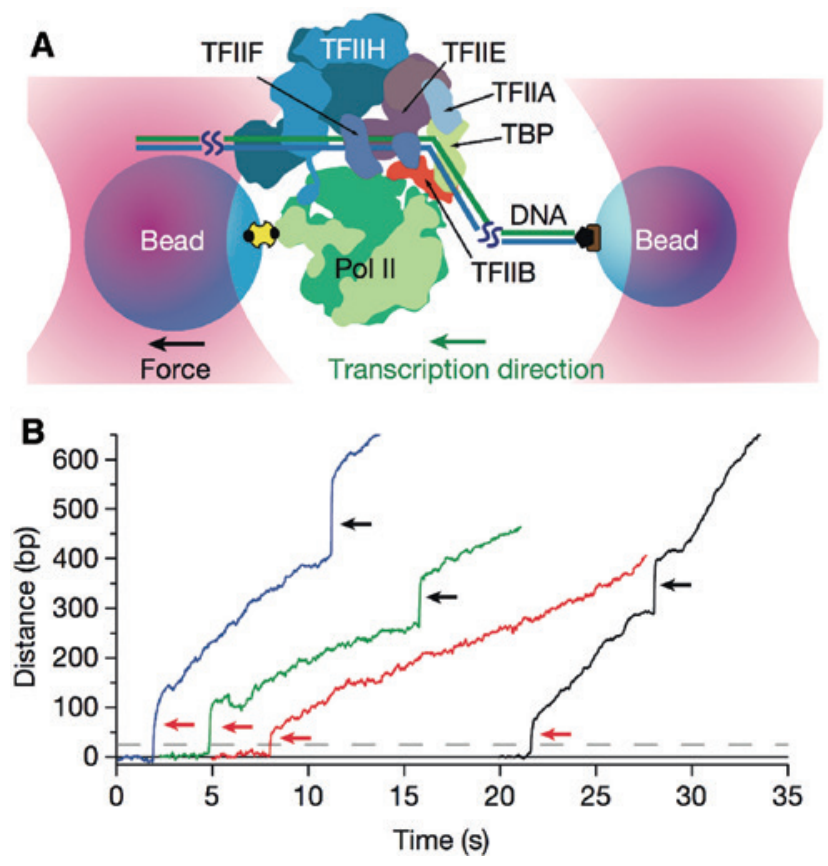

Figure 2: Transcription initiation by Pol II in assisting force mode. (A) Schematic diagram of the transcription initiation assisting force mode set-up, where a tether is formed between the two beads (blue), with Pol II attached to one bead, and the upstream DNA attached to the second bead. As transcription proceeds, the distance between the beads increases. All proteins used for initiation are depicted here, except Sub1. The scheme is not to scale. (B) Representative traces of Pol II elongation, where red arrows mark the promotor escape and the black arrows mark the increase of force that was used to confirm elongation. Adapted from Ref. (10) with permission from Nature Publishing Group.

investigated elongation rates and forces at which the polymerase stops transcribing. The velocity of Escherichia coli RNAP was measured to be $16 \mathrm{nt} / \mathrm{s}$. Interestingly, the velocity of the polymerase was uniform until the stalling force was reached, on average at $14 \mathrm{pN}$ (31). Later studies found that RNAP can transcribe up to $25 \mathrm{pN}$ of force (32).

Optical tweezers elongation studies of a eukaryotic polymerase, Pol II, showed that this enzyme can transcribe as fast as $12 \mathrm{nt} / \mathrm{s}$, but to lower opposing forces than the bacterial one, around $7.5 \mathrm{pN}$ (6). The velocity of Pol II was reported in many later optical tweezers studies. Depending on the experimental system, i.e. assisting or opposing force mode and the concentration of nucleotides used, the measured velocity ranged between 15 and $25 \mathrm{nt} / \mathrm{s}(9,11,21,22,33-35)$.

A recent optical tweezers study of another eukaryotic RNA polymerase, Pol I, reported a pause-free velocity of $39 \mathrm{nt} / \mathrm{s}$ in assisting and $31 \mathrm{nt} / \mathrm{s}$ in opposing force mode (11). Comparison of yeast Pol I and Pol II pause-free velocities showed that Pol I is around 1.5 times faster. This is consistent with structural studies that identified Pol I specific subunits that stimulate overall elongation. The subcomplex A49/A34.5, homologous to the Pol II transcription factor TFIIF $(36,37)$, was found to stimulate Pol I elongation as shown with bulk RNA extension assays (38).

\section{The nucleotide addition cycle}

During elongation, in each nucleotide addition cycle (NAC), the polymerase has to bind the correct nucleoside triphosphate (NTP), form a bond between the new NTP and the 3 '-end of the RNA, release the pyrophosphate (PPi) and translocate one base pair to make space for the next NTP (39). However, the enzyme does not only move forward, but sometimes pauses and moves backwards along the DNA template (see next section). Both forward and backward steps have to be finely tuned and happen at a rate that ensures adequate speed and fidelity of transcription elongation.

Several theoretical models that describe NAC were proposed in the past, including a 'power-stroke' model that assumes that a conformational change of the enzyme coupled with NTP hydrolysis drives the enzyme forward [reviewed in Ref. (40)]. However, today, it is generally accepted that the NAC can be explained by a 'Brownian ratchet' theoretical model, in which the polymerase oscillates between the pre- and the post-translocated states and is driven forward by the incoming nucleotide (7, 21, 41-44).

Several optical tweezers studies attempted to characterise the kinetics of the NAC. Larson and colleagues analysed how force affects pause-free velocity (obtained by removing pause regions from the transcription traces) of Pol II (44). If one assumes that the translocation step of the polymerase is fast, and the rate-limiting step is the NTP catalysis, then the force-velocity relationship would eventually plateau as the NTP concentration increases, because the enzyme would spend less time in the forcesensitive translocation phase. However, this was not seen in the experimental data. Therefore, Larson and colleagues proposed that there must exist two binding sites for the incoming NTP, and that the incoming NTP can bind the polymerase in both the pre-translocated and the posttranslocated state. Furthermore, using trigger loop Pol II mutants, they showed that the trigger loop structural element affects NTP binding, enzyme translocation and catalysis.

Dangulkwanich and colleagues analysed forcevelocity relationship and pausing dynamics of Pol II with varying NTP concentrations, under assisting and opposing 
forces, and on nucleosomal and bare DNA template (21). By combining experimental data and theoretical modelling, they extracted forward translocation and pausing rates. Surprisingly, they found that the rate of translocation of the enzyme is of the same order as the catalysis rate. The authors suggested that the slower translocation rate is sufficient to explain the force-velocity relationship of the enzyme, and therefore discarded the need of a more complicated model that includes two NTP binding sites.

\section{Pausing of RNA polymerases}

Pausing represents an off-pathway from the nucleotide addition cycle that enables many co-transcriptional processes, such as promotor-proximal pausing of Pol II and co-transcriptional pre-mRNA splicing (45-47). The most prominent mechanism of pausing is backtracking (48, 49). Backtracking involves movement of the polymerase in the upstream direction on the DNA template, resulting in displacement of the $3^{\prime}$-end of the RNA from the active site (50-52). Backtracking is widespread in vivo, occurring frequently throughout the entire length of the genes in yeast and at the $3^{\prime}$-end of exons in human cells $(53,54)$, and it was also detected in optical tweezers experiments $(9,11,21,55,56)$. Backtracking is essential for proofreading and for overcoming obstacles such as nucleosomes $(53,57-60)$.

In the backtracked state, the polymerase performs a one-dimensional random walk along the DNA template (6, 61). The backtrack is recovered when the polymerase realigns the 3'-end of the RNA in the active site and continues to transcribe $(21,22,35,55,56,60,61)$ or when the backtracked RNA is cleaved off, which generates a new 3'-end in the active site $(38,62,63)$. Cleavage can be performed intrinsically by the enzymes or, in the Pol II system, it can be assisted by a transcription factor, TFIIS (64-66).

Several factors were shown to influence the entry of a polymerase into a backtrack and the duration of backtracking. It was proposed that the secondary structure of the transcribed RNA presents an energetic barrier for polymerase backtracking, thus preventing the polymerase to go into a deep backtrack and reducing the overall backtracking time (67). Theoretical predictions were confirmed experimentally in an optical tweezers study that showed that the GC content of the DNA template and hence, the nascent RNA structure, affects the pausing dynamics of RNA polymerases, with polymerases pausing less on the GC-rich templates that are known to form more secondary structures (34). The energy barrier to backtracking due to the secondary structure of the RNA is also considered in a recent theoretical study, where the entropy produced during backtracking is discussed (68). The authors derived relations for the probabilities of backward and forward stepping of a backtracked RNA polymerase as well as bounds for the distribution of the maximum backtrack depth. Furthermore, it was shown that the relative instability of the RNA : DNA hybrid causes the polymerase to slide backwards (69). Interestingly, the coding regions of the genes, exons, show higher RNA : DNA hybrid stability $(70,71)$, suggesting the possibility of different pausing dynamics between exons and introns.

Backtracking has been modelled previously as a onedimensional (1D) random walk in discrete $(21,22,35,44,60$, 61) or continuous space $(11,56,72)$. Depken and colleagues modelled backtracking as a 1D hopping process, where the duration of the backtrack corresponds to a first-passage time of the random walker (61). The authors focused on verifying whether backtracking can also explain the short, ubiquitous pauses detected during elongation (73, 74). Their work showed that a backtracking mechanism can give rise to both short pauses that are exponentially distributed and intermediate and long pauses that follow a $t^{-3 / 2}$ power law. Later studies questioned the validity of a purely diffusive model of backtracking. Schweikhard and colleagues showed that short pauses can be equally well explained with different models, such as, power-law, diffusion and a multi-exponential model (9). Indeed, for long pauses the statistics that are accessible in experiments often are low. Hence, additional mechanisms intrinsic to Pol II that modulate the enzyme backtracking have been proposed, such as the existence of a 'gating tyrosine' which allows $1 \mathrm{bp}$ backtracks but restricts further backtracking (52). To conclude, pausing during transcription is a complex process that might very well be dominated by backtracking, but it is also possible that other mechanisms contribute significantly.

Finally, to continue transcribing, a backtracked RNA polymerase has to eventually recover from the backtracked state. A recent study from our group investigated the backtrack recovery of Pol I and Pol II (11). Our work showed that Pol I and Pol II use different strategies to recover from different backtrack depths: short backtracks are recovered by 1D diffusion while intermediate ones recover by transcript cleavage. Interestingly, Pol I and Pol II have comparable diffusion rates, therefore differences in backtrack recovery dynamics stem mainly from differences in cleavage activities. Modelling the polymerase backtrack recovery as a stochastic resetting process showed that the choice of a recovery pathway is determined by a kinetic competition between $1 \mathrm{D}$ diffusion and cleavage $(11,72)$. 


\section{Transcription through nucleosomes}

The genetic material of eukaryotic cells is organised into chromatin, a complex of DNA wrapped around histone proteins forming nucleosomes. It was proposed by bulk experiments that nucleosomes represent an energetic barrier to elongating Pol II (75-77). Several optical tweezers studies further investigated how Pol II overcomes nucleosomes during elongation and successfully transcribes eukaryotic genes.

Hodges and colleagues used optical tweezers to study transcription of Pol II through a single nucleosome template (Figure 3A) (22). Their studies confirmed that a nucleosome behaves like a barrier that both decreases the rate of forward translocation of Pol II and increases pausing and backtracking. Furthermore, they found that the progression of the enzyme through the nucleosome depends on nucleosomal fluctuations, i.e. nucleosomal breathing or local unwrapping of the nucleosomal DNA due to thermal fluctuations. The nucleosome unwraps the DNA locally, thereby giving the polymerase enough DNA to transcribe through. Bintu and colleagues further investigated the influence of specific histone modifications and nascent RNA secondary structure on transcription through nucleosomes (23). They showed that the local nucleosome unwrapping dynamics, and in turn the transcription elongation dynamics, is affected by nucleosomal properties such as the histones modification state, presence of histone tails or the underlying DNA sequence.

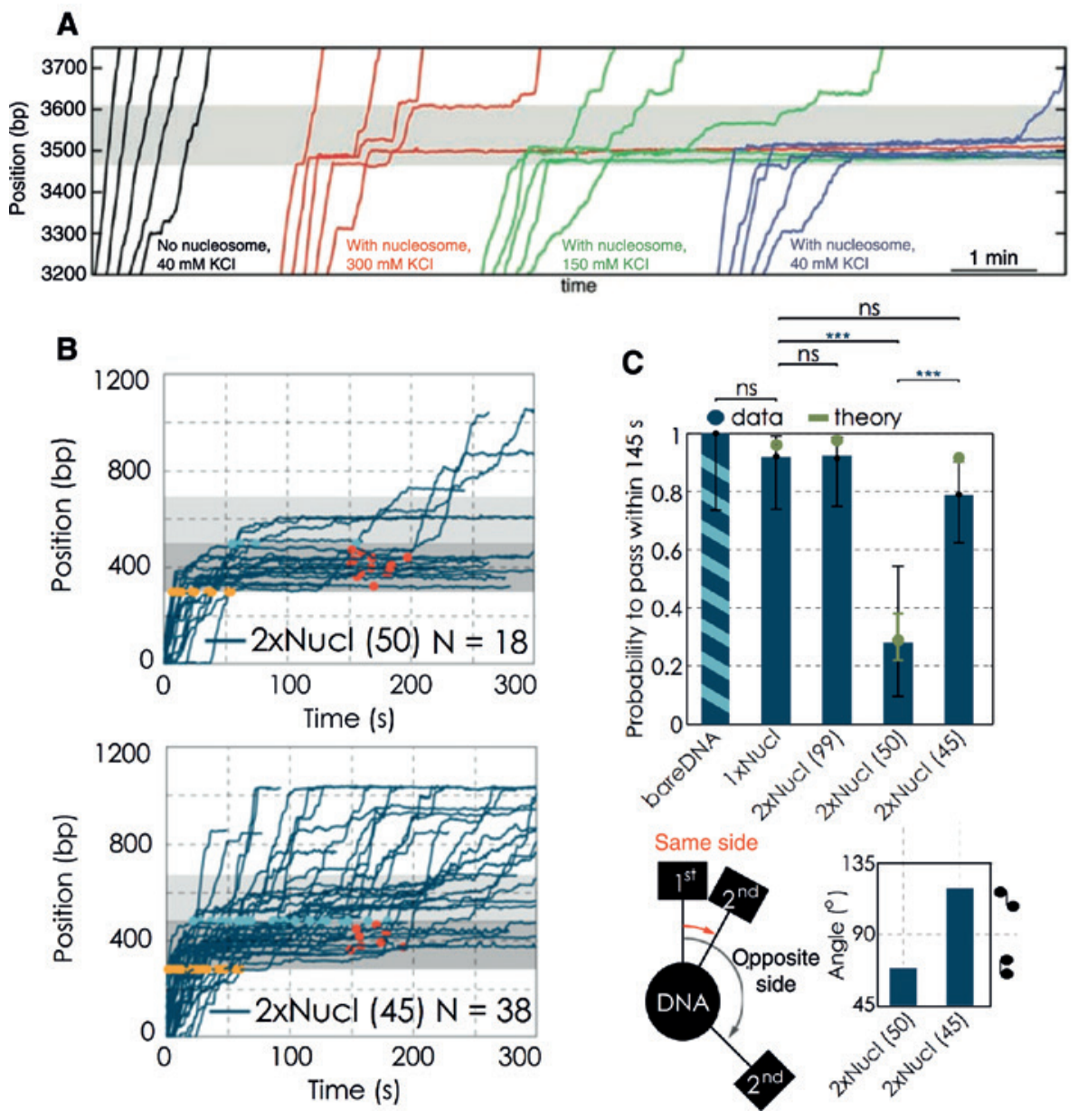

Figure 3: Transcription through nucleosomal DNA.

(A) Transcription traces of individual Pol Il transcribing through a single nucleosome template with different ionic strengths. The presence of a nucleosome represents a barrier to transcription, causing long pauses and arrest of Pol II. (B) Transcription traces of individual Pol II transcribing through dinucleosomal DNA templates, at $50 \mathrm{bp}$ and $45 \mathrm{bp}$ distance. The presence of a second nucleosome at a $50 \mathrm{bp}$ distance from the first one impairs Pol II transcription dynamics through the first nucleosome. (C) (Upper) Passage probabilities of Pol II through the first nucleosomal region: Pol II transcribes more efficiently through two nucleosomes at 45-bp than at 50-bp distance. (Lower) Angular distributions of the two neighbouring nucleosomes for the 50-bp (nucleosomes face the same side of the DNA) and 45-bp (nucleosomes face opposite sides of the DNA) linker templates. Adapted from Refs. $(22,24)$ with permission from AAAS and USA National Academy of Sciences. 
Recently, our group used optical tweezers to study transcription of Pol II through nucleosomal templates of higher complexity and the influence of nucleosomal geometry on the efficiency of transcription (Figure 3B) (24). Fitz and colleagues investigated Pol II elongation dynamics along a DNA template with two nucleosomes. Our work showed that the presence of a second nucleosome affects the probability of the polymerase to pass through the first nucleosome. This effect is dependent on the spacing between the two nucleosomes and their rotational arrangement along the DNA (Figure 3C). Pol II could transcribe more efficiently when the second nucleosome was facing opposite side of the DNA template, suggesting that the 3D nucleosomal arrangement affects Pol II transcription dynamics.

\section{Elongation with transcription factors}

The dynamics of transcription elongation is regulated by different transcription factors, such as TFIIS and TFIIF in the case of eukaryotic Pol II. The elongation factors act through various mechanisms, increasing overall transcription rates or by affecting proofreading and fidelity and by reactivating backtracked polymerases. Optical tweezers assays are particularly suitable for studying the influence of individual transcription factors on polymerase elongation, as they allow timely-controlled addition of purified factors to the transcribing elongation complex.

One of the most studied transcription factor in optical tweezers assays is TFIIS. This protein is known to enhance the cleavage of backtracked RNA and recovery of backtracked Pol II $(9,11,35,50,52,53,56,64)$. Without TFIIS, Pol II can only transcribe up to forces of $7.5 \mathrm{pN}$, before entering a backtrack. However, by rescuing backtracked polymerases, TFIIS enables Pol II to transcribe up to 16.9 pN (Figure 4A) (56). Therefore, TFIIS enables Pol II to transcribe against higher loads, increasing its mechanical performance, which in turn allows it to transcribe through nucleosomes more efficiently (35). TFIIS acts mainly by affecting the pausing dynamics of Pol II, decreasing the time the enzyme spends in a pause and not by affecting its overall transcription rate (35). Two different studies investigated the dynamics of TFIIS-assisted reactivation of backtracked Pol II $(9,11)$. By using a cleavage-defective mutant, Schweikhard and colleagues showed that at low force TFIIS can relieve arrested Pol II in a cleavageindependent way. The authors argued that this might be in accordance with structural results that show that TFIIS weakens the interactions of backtracked RNA and Pol II, by competing for the same binding sites inside the Pol II funnel (52). Furthermore, the work from our group showed that TFIIS increases the backtrack recovery efficiency of Pol II. TFIIS enables rapid recovery from backtracks of any depth and even when it associates with an already backtracked enzyme (11).

Another transcription factor affecting the dynamics of Pol II elongation is TFIIF. Apart from being a general transcription initiation factor, TFIIF was shown to enhance overall transcription rates of Pol II $(78,79)$. Optical tweezers experiments confirmed that TFIIF enhances elongation efficiency of Pol II (Figure 4B), but not through changing pause-free velocity of the enzyme, but by decreasing the frequency and the duration of Pol II pausing during assisting force mode assays (35). Furthermore, Schweikhard and colleagues found that TFIIF stimulates the restart of the backtracked Pol II in a lowforce regime. However, at high forces there is no effect of TFIIF alone on Pol II backtrack recovery, although it
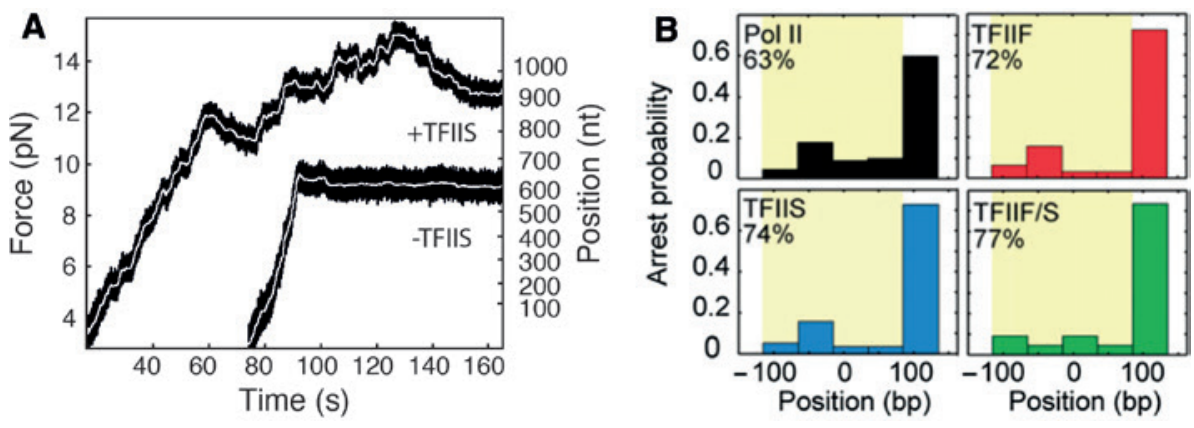

Figure 4: Elongation in the presence of transcription factors.

(A) Transcription traces of Pol II in the presence and absence of TFIIS. TFIIS rescues backtracked Pol II and enables it to transcribe to higher forces. (B) Arrest probabilities of Pol II in a nucleosome region (marked in yellow), in the presence of TFIIS, TFIIF or both. Both TFIIS and TFIIF enable Pol II to transcribe more efficiently through nucleosomes, as seen with the increased percentage of passages through the nucleosome region. Adapted from Refs. $(11,35)$ with permission from USA National Academy of Sciences. 
enhances the effects of TFIIS. The authors argued that TFIIF could interact with TFIIS and in this way stabilise it on the backtracked Pol II complex.

\section{Termination}

Termination is the final stage of transcription during which a transcribing polymerase and the nascent RNA are released from the DNA. Termination defines boundaries of transcription units, ensuring there is no interfering with the transcription of the downstream gene and securing there is enough RNA polymerase ready for reinitiation. Termination in prokaryotes can be mediated through terminator sequences within the nascent RNA that form a stem-loop structure and cause the RNA polymerase to stop and dissociate; or through a Rho-dependent mechanism, in which a Rho protein binds to the RNA and runs towards the RNAP (80). Termination mechanisms in eukaryotes are more complex and remain poorly understood (81-84). Therefore, optical tweezers studies thus far focused only on prokaryotic termination.

In both mechanisms of prokaryotic transcription termination, the release of the polymerase is caused by the forces exerted on the RNA, either by folding of the RNA hairpin or by Rho displacement, which results in forward translocation of the polymerase without the RNA synthesis. Therefore, optical tweezers studies focused on applying external forces on the nascent RNA. Dalal and colleagues performed experiments with an experimental geometry that allows pulling on the elongating RNA chain (8). They found that RNAP could transcribe up to forces of $30 \mathrm{pN}$ applied to the RNA, which demonstrates high TEC stability and implies that loads required to terminate elongation have to be higher than this level. Larson and colleagues used similar RNA and DNA pulling assay to investigate the transcription efficiency of different terminator sequences that differ by the size of the hairpin and the length of the U-tract (85). They found that termination occurs through a combination of shearing and forward translocation mechanisms, depending on the composition of the U-track of the terminator sequence.

A recent study by Koslover and colleagues used optical tweezers to investigate the interaction of the Rho protein with the nascent RNA (86). The authors used a dumbell assay that consisted of an RNAP that was attached to the surface of a streptavidin bead and the nascent RNA that was annealed to a DNA handle, which was attached to another bead. In this configuration, the Rho protein binds to the nascent RNA and in the presence of ATP translocates towards the RNAP. Detachment of Rho from the RNA was seen as a rip in the force-extension curves. They found that Rho can adopt two RNA binding states, one that binds around $57 \mathrm{nt}$ and one that binds $85 \mathrm{nt}$. Furthermore, they suggested that Rho translocates towards the RNAP by a tethered-tracking mechanism, looping out the RNA between Rho and the RNAP. The assay is suitable for further studies of transcription termination, studying the step size of Rho protein and the interaction with its cofactors, NusA and NusG.

\section{Conclusions and outlook}

Optical tweezers studies have in the last two decades revealed numerous micromechanical details of the transcription process. They have proven to be an invaluable tool to study gene transcription. Initially, optical tweezers studies investigated viral or bacterial enzymes running on the DNA template. However, over the years, they have become increasingly more complex, probing the functions of different eukaryotic RNA polymerases, numerous transcription factors and considering complex 3D DNA/nucleosome arrangements. A key feature of all these studies lies in the fact that optical tweezers measure actual trajectories of individual enzymes, providing a wealth of details on the dynamics of the process. The quantitative nature of optical tweezers data makes it an ideal tool for testing various theoretical models of translocation, pausing, and recently as an example of a stochastic resetting process (72). On the side of physics, the implications of utilizing a template for polymerizing a copolymer (RNA) on the dynamics of the process are just beginning to become grasped (87). Furthermore, as theoretical models become more and more refined, the amount of single molecule data required for comparing and distinguishing between them increases. New developments are needed on the experimental side in order to achieve this, perhaps by switching to fully automated optical tweezer experiments.

However, many questions remain open. The largest eukaryotic RNA polymerase, RNA polymerase III, has not been studied with optical tweezers. Comparing transcription dynamics of the three eukaryotic polymerases will provide insights into the evolution of these enzymes. In particular, the cleavage activity of the three eukaryotic polymerases was shown to be differently regulated (66). Obtaining data on backtracking dynamics and recovery of Pol III would provide further insights into the nature of transcriptional pausing in eukaryotes. Much of the transcription studies thus far have been performed on plasmid 
DNA. It would be interesting to use actual gene templates in the future, to investigate the influence of the underlying sequence and gene features on transcription dynamics.

Finally, future studies of transcription will greatly benefit from combining optical tweezers with other techniques. In combination with fluorescence microscopy, optical tweezers were used in DNA overstretching experiments and for determining DNA-protein interactions (8890). Angular optical trapping (AOT) was developed for simultaneous detection of force and torque $(91,92)$, which enabled studying transcription dynamics and nucleosome stability under conditions of applied torque on the DNA (93). Recent advancements in combining optical tweezers with confocal microscopy, as well as development of advanced microfluidics systems will likely increase the range of applications and enable investigation of conditions more similar to the in vivo situation.

To conclude, much has been learned, but we are still far from a detailed understanding of how the information that is contained within DNA is translated to RNA form. Optical tweezers studies will contribute to the future advances in the field.

Acknowledgements: We would like to thank Édgar Roldán for his suggestions on the manuscript. S.W.G. was supported by the DFG (SPP 1782, GSC 97, GR 3271/2, GR 3271/3, GR 3271/4), the European Research Council (grant No. 281903), ITN grants 281903 and 641639 from the EU, the Max-Planck-Society as a Max-Planck-Fellow, and the Human Frontier Science Program (RGP0023/2014).

\section{List of abbreviations}

$\begin{array}{ll}\text { AOT } & \text { angular optical trapping } \\ \text { DNA } & \text { deoxyribonucleic acid } \\ \text { E. coli } & \text { Escherichia coli } \\ \text { FRET } & \text { Förster resonance energy transfer } \\ \text { NAC } & \text { nucleotide addition cycle } \\ \text { NTP } & \text { nucleoside triphosphate } \\ \text { PIC } & \text { pre-initiation complex } \\ \text { Pol II } & \text { RNA polymerase II } \\ \text { PPi } & \text { pyrophosphate } \\ \text { pre-mRNA } & \text { pre-messenger RNA } \\ \text { RNA } & \text { ribonucleic acid } \\ \text { RNAPs } & \text { RNA polymerases } \\ \text { TEC } & \text { transcription elongation complex }\end{array}$

\section{References}

1. Cramer P, Armache KJ, Baumli S, Benkert S, Brueckner F, Buchen C, Damsma GE, Dengl S, Geiger SR, Jasiak AJ, Jawhari A.
Structure of eukaryotic RNA polymerases. Annu Rev Biophys 2008; 37: 337-52.

2. Jonkers I, Lis JT. Getting up to speed with transcription elongation by RNA polymerase II. Nat Rev Mol Cell Biol 2015; 16: 167-77.

3. Darzacq X, Yao J, Larson DR, Causse SZ, Bosanac L, De Turris V, Ruda VM, Lionnet T, Zenklusen D, Guglielmi B, Tjian R. Imaging transcription in living cells. Annu Rev Biophys 2009; 38: 173.

4. Bustamante C, Cheng W, Mejia YX. Revisiting the central dogma one molecule at a time. Cell 2011; 144: 480-97.

5. Larson MH, Landick R, Block SM. Single-molecule studies of RNA polymerase: one singular sensation, every little step it takes. Mol Cell 2011; 41: 249-62.

6. Galburt EA, Grill SW, Bustamante C. Single molecule transcription elongation. Methods 2009; 48: 323-32.

7. Abbondanzieri EA, Greenleaf WJ, Shaevitz JW, Landick R, Block SM. Direct observation of base-pair stepping by RNA polymerase. Nature 2005; 438: 460-5.

8. Dalal RV, Larson MH, Neuman KC, Gelles J, Landick R, Block SM. Pulling on the nascent RNA during transcription does not alter kinetics of elongation or ubiquitous pausing. Mol Cell 2006; 23: 231-9.

9. Schweikhard V, Meng C, Murakami K, Kaplan CD, Kornberg RD, Block SM. Transcription factors TFIIF and TFIIS promote transcript elongation by RNA polymerase II by synergistic and independent mechanisms. Proc Natl Acad Sci USA 2014; 111: 6642-7.

10. Fazal FM, Meng CA, Murakami K, Kornberg RD, Block SM. Realtime observation of the initiation of RNA polymerase II transcription. Nature 2015; 525: 274-7.

11. Lisica A, Engel C, Jahnel M, Roldán É, Galburt EA, Cramer P, Grill SW. Mechanisms of backtrack recovery by RNA polymerases I and II. Proc Natl Acad Sci USA 2016; 113: 2946-51.

12. Fazal FM, Block SM. Optical tweezers study life under tension. Nat Photonics 2011; 5: 318-21.

13. Ashkin A. Acceleration and trapping of particles by radiation pressure. Phys Rev Lett 1970; 24: 156.

14. Ashkin A, Dziedzic JM, Bjorkholm JE, Chu S. Observation of a single-beam gradient force optical trap for dielectric particles. Optics Lett 1986; 11: 288-90.

15. Neuman KC, Block SM. Optical trapping. Rev Sci Instr 2004; 75: 2787-809.

16. Berg-Sørensen K, Flyvbjerg H. Power spectrum analysis for optical tweezers. Rev Sci Instr 2004; 75: 594-612.

17. Van Mameren J, Peterman EJ, Wuite GJ. See me, feel me: methods to concurrently visualize and manipulate single DNA molecules and associated proteins. Nucleic Acids Res 2008; 36: 4381-9.

18. Smith SB, Cui Y, Bustamente C. Overstretching B-DNA: the elastic response of individual double-stranded and single-stranded DNA molecules. Science 1996; 271: 795.

19. Wang MD, Yin H, Landick R, Gelles J, Block SM. Stretching DNA with optical tweezers. Biophys J 1997; 72: 1335.

20. Wuite GJ, Smith SB, Young M, Keller D, Bustamante C. Singlemolecule studies of the effect of template tension on T7 DNA polymerase activity. Nature 2000; 404: 103-6.

21. Dangkulwanich M, Ishibashi T, Liu S, Kireeva ML, Lubkowska L, Kashlev M, Bustamante CJ. Complete dissection of transcription elongation reveals slow translocation of RNA polymerase II in a linear ratchet mechanism. eLife 2013; 2: e00971. 
22. Hodges C, Bintu L, Lubkowska L, Kashlev M, Bustamante C. Nucleosomal fluctuations govern the transcription dynamics of RNA polymerase II. Science 2009; 325: 626-8.

23. Bintu L, Ishibashi T, Dangkulwanich M, Wu YY, Lubkowska L, Kashlev M, Bustamante C. Nucleosomal elements that control the topography of the barrier to transcription. Cell 2012; 151: 738-49.

24. Fitz V, Shin J, Ehrlich C, Farnung L, Cramer P, Zaburdaev V, Grill SW. Nucleosomal arrangement affects single-molecule transcription dynamics. Proc Natl Acad Sci USA 2016; 45: 12733-8.

25. Conaway RC, Conaway JW. General initiation factors for RNA polymerase II. Annu Rev Biochem 1993; 62: 161-90.

26. Kapanidis AN, Margeat E, Ho SO, Kortkhonjia E, Weiss S, Ebright $\mathrm{RH}$. Initial transcription by RNA polymerase proceeds through a DNA-scrunching mechanism. Science 2006; 314: 1144-7.

27. Revyakin A, Liu C, Ebright RH, Strick TR. Abortive initiation and productive initiation by RNA polymerase involve DNA scrunching. Science 2006; 314: 1139-43.

28. Kapanidis AN, Margeat E, Laurence TA, Doose S, Ho SO, Mukhopadhyay J, Kortkhonjia E, Mekler V, Ebright RH, Weiss S. Retention of transcription initiation factor $\mathrm{s} 70$ in transcription elongation: single-molecule analysis. Mol Cell 2005; 20: 347-56.

29. Chakraborty A, Wang D, Ebright YW, Korlann Y, Kortkhonjia E, Kim T, Chowdhury S, Wigneshweraraj S, Irschik H, Jansen R, Nixon BT. Opening and closing of the bacterial RNA polymerase clamp. Science 2012; 337: 591-5.

30. Duchi D, Bauer DL, Fernandez L, Evans G, Robb N, Hwang LC, Gryte K, Tomescu A, Zawadzki P, Morichaud Z, Brodolin K. RNA polymerase pausing during initial transcription. Mol Cell 2016; 63: 939-50.

31. Yin H, Wang MD, Svoboda K, Landick R. Transcription against an applied force. Science 1995; 270: 1653.

32. Wang MD, Schnitzer MJ, Yin H, Landick R, Gelles J, Block SM. Force and velocity measured for single molecules of RNA polymerase. Science 1998; 282: 902-7.

33. Larson DR, Zenklusen D, Wu B, Chao JA, Singer RH. Real-time observation of transcription initiation and elongation on an endogenous yeast gene. Science 2011; 332: 475-8.

34. Zamft B, Bintu L, Ishibashi T, Bustamante C. Nascent RNA structure modulates the transcriptional dynamics of RNA polymerases. Proc Natl Acad Sci USA 2012; 109: 8948-53.

35. Ishibashi T, Dangkulwanich M, Coello Y, Lionberger TA, Lubkowska L, Ponticelli AS, Kashlev M, Bustamante C. Transcription factors IIS and IIF enhance transcription efficiency by differentially modifying RNA polymerase pausing dynamics. Proc Natl Acad Sci USA 2014; 111: 3419-24.

36. Engel C, Sainsbury S, Cheung AC, Kostrewa D, Cramer P. RNA polymerase I structure and transcription regulation. Nature 2013; 502: 650-5.

37. Fernández-Tornero C, Moreno-Morcillo M, Rashid UJ, Taylor NM, Ruiz FM, Gruene T, Legrand P, Steuerwald U, Müller CW. Crystal structure of the 14-subunit RNA polymerase I. Nature 2013; 502: 644-9.

38. Kuhn CD, Geiger SR, Baumli S, Gartmann M, Gerber J, Jennebach $\mathrm{S}$, Mielke T, Tschochner H, Beckmann R, Cramer P. Functional architecture of RNA polymerase I. Cell 2007; 131: 1260-72.

39. Erie DA, Yager TD, Von Hippel PH. The single-nucleotide addition cycle in transcription: a biophysical and biochemical perspective. Annu Rev Biophys Biomol Struct 1992; 21: 379-415.
40. Zhou J, Schweikhard V, Block SM. Single-molecule studies of RNAPII elongation. Biochim Biophys Acta Gene Regul Mech 2013; 1829: 29-38.

41. Guajardo R, Sousa R. A model for the mechanism of polymerase translocation. J Mol Biol 1997; 265: 8-19.

42. Komissarova N, Kashlev M. RNA polymerase switches between inactivated and activated states by translocating back and forth along the DNA and the RNA. J Biol Chem 1997; 272: 15329-38.

43. Bar-Nahum G, Epshtein V, Ruckenstein AE, Rafikov R, Mustaev A, Nudler $E$. A ratchet mechanism of transcription elongation and its control. Cell 2005; 120: 183-93.

44. Larson MH, Zhou J, Kaplan CD, Palangat M, Kornberg RD, Landick R, Block SM. Trigger loop dynamics mediate the balance between the transcriptional fidelity and speed of RNA polymerase II. Proc Natl Acad Sci USA 2012; 109: 6555-60.

45. Muse GW, Gilchrist DA, Nechaev S, Shah R, Parker JS, Grissom SF, Zeitlinger J, Adelman K. RNA polymerase is poised for activation across the genome. Nat Genet 2007; 39: 1507-11.

46. Oesterreich FC, Preibisch S, Neugebauer KM. Global analysis of nascent RNA reveals transcriptional pausing in terminal exons. Mol Cell 2010; 40: 571-81.

47. Alexander RD, Innocente SA, Barrass JD, Beggs JD. Splicingdependent RNA polymerase pausing in yeast. Mol Cell 2010; 40: 582-93.

48. Nudler E, Mustaev A, Goldfarb A, Lukhtanov E. The RNA-DNA hybrid maintains the register of transcription by preventing backtracking of RNA polymerase. Cell 1997; 89: 33-41.

49. Komissarova N, Kashlev M. Transcriptional arrest: Escherichia coli RNA polymerase translocates backward, leaving the 3' end of the RNA intact and extruded. Proc Natl Acad Sci USA 1997; 94 : 1755-60.

50. Kettenberger H, Armache KJ, Cramer P. Architecture of the RNA polymerase II-TFIIS complex and implications for mRNA cleavage. Cell 2003; 114: 347-57.

51. Wang D, Bushnell DA, Huang X, Westover KD, Levitt M, Kornberg RD. Structural basis of transcription: backtracked RNA polymerase II at $3.4 \AA$ A resolution. Science 2009; 324: 1203-6.

52. Cheung AC, Cramer P. Structural basis of RNA polymerase II backtracking, arrest and reactivation. Nature 2011; 471: 249-53.

53. Churchman LS, Weissman JS. Nascent transcript sequencing visualizes transcription at nucleotide resolution. Nature 2011; 469: 368-73.

54. Mayer A, Di lulio J, Maleri S, Eser U, Vierstra J, Reynolds A, Sandstrom R, Stamatoyannopoulos JA, Churchman LS. Native elongating transcript sequencing reveals human transcriptional activity at nucleotide resolution. Cell 2015; 161: 541-54.

55. Shaevitz JW, Abbondanzieri EA, Landick R, Block SM. Backtracking by single RNA polymerase molecules observed at near-basepair resolution. Nature 2003; 426: 684-7.

56. Galburt EA, Grill SW, Wiedmann A, Lubkowska L, Choy J, Nogales E, Kashlev M, Bustamante C. Backtracking determines the force sensitivity of RNAP II in a factor-dependent manner. Nature 2007; 446: 820-3.

57. Erie DA, Hajiseyedjavadi O, Young MC, Von Hippel PH. Multiple RNA polymerase conformations and GreA: control of the fidelity of transcription. Science 1993; 262: 867-73.

58. Thomas MJ, Platas AA, Hawley DK. Transcriptional fidelity and proofreading by RNA polymerase II. Cell 1998; 93: 627-37.

59. Sydow JF, Brueckner F, Cheung AC, Damsma GE, Dengl S, Lehmann E, Vassylyev D, Cramer P. Structural basis of 
transcription: mismatch-specific fidelity mechanisms and paused RNA polymerase II with frayed RNA. Mol Cell 2009; 34: 710-21.

60. Depken M, Parrondo JM, Grill SW. Intermittent transcription dynamics for the rapid production of long transcripts of high fidelity. Cell Rep 2013; 5: 521-30.

61. Depken M, Galburt EA, Grill SW. The origin of short transcriptional pauses. Biophys I 2009; 96: 2189-93.

62. Walmacq C, Kireeva ML, Irvin J, Nedialkov Y, Lubkowska L, Malagon F, Strathern JN, Kashlev M. Rpb9 subunit controls transcription fidelity by delaying NTP sequestration in RNA polymerase II. J Biol Chem 2009; 284: 19601-12.

63. Chédin S, Riva M, Schultz P, Sentenac A, Carles C. The RNA cleavage activity of RNA polymerase III is mediated by an essential TFIIS-like subunit and is important for transcription termination. Genes Dev 1998; 12: 3857-71.

64. Izban MG, Luse DS. The RNA polymerase II ternary complex cleaves the nascent transcript in a 3'?5'direction in the presence of elongation factor SII. Genes Dev 1992; 6: 1342-56.

65. Fish RN, Kane CM. Promoting elongation with transcript cleavage stimulatory factors. Biochim Biophys Acta Gene Struct Expr 2002; 1577: 287-307.

66. Ruan W, Lehmann E, Thomm M, Kostrewa D, Cramer P. Evolution of two modes of intrinsic RNA polymerase transcript cleavage. J Biol Chem 2011; 286: 18701-7.

67. Klopper AV, Bois JS, Grill SW. Influence of secondary structure on recovery from pauses during early stages of RNA transcription. Phys Rev E 2010; 81: 030904.

68. Neri I, Roldán É, Jülicher F. Statistics of infima and stopping times of entropy production and applications to active molecular processes. Pays Rev X 2017; (in press). arXiv:1604.04159.

69. Palangat M, Landick R. Roles of RNA: DNA hybrid stability, RNA structure, and active site conformation in pausing by human RNA polymerase II. J Mol Biol 2001; 311: 265-82.

70. Kraeva RI, Krastev DB, Roguev A, Ivanova A, Nedelcheva-Veleva MN, Stoynov SS. Stability of mRNA/DNA and DNA/DNA duplexes affects mRNA transcription. PLoS One 2007; 2: e290.

71. Nedelcheva-Veleva MN, Sarov M, Yanakiev I, Mihailovska E, Ivanov MP, Panova GC, Stoynov SS. The thermodynamic patterns of eukaryotic genes suggest a mechanism for intron-exon recognition. Nat Commun 2013; 4: 2101.

72. Roldán É, Lisica A, Sánchez-Taltavull D, Grill SW. Stochastic resetting in backtrack recovery by RNA polymerases. Phys Rev E 2016; 93: 062411.

73. Neuman KC, Abbondanzieri EA, Landick R, Gelles J, Block SM. Ubiquitous transcriptional pausing is independent of RNA polymerase backtracking. Cell 2003; 115: 437-47.

74. Herbert KM, La Porta A, Wong BJ, Mooney RA, Neuman KC, Landick R, Block SM. Sequence-resolved detection of pausing by single RNA polymerase molecules. Cell 2006; 125: 1083-94.

75. Izban MG, Luse DS. Transcription on nucleosomal templates by RNA polymerase II in vitro: inhibition of elongation with enhancement of sequence-specific pausing. Genes Dev 1991; 5: 683-96.

76. Kireeva ML, Walter W, Tchernajenko V, Bondarenko V, Kashlev M, Studitsky VM. Nucleosome remodeling induced by RNA polymerase II: loss of the $\mathrm{H} 2 \mathrm{~A} / \mathrm{H} 2 \mathrm{~B}$ dimer during transcription. Mol Cell 2002; 9: 541-52.

77. Bondarenko VA, Steele LM, Újvári A, Gaykalova DA, Kulaeva OI, Polikanov YS, Luse DS, Studitsky VM. Nucleosomes can form a polar barrier to transcript elongation by RNA polymerase II. Mol Cell 2006; 24: 469-79.

78. Izban MG, Luse DS. Factor-stimulated RNA polymerase II transcribes at physiological elongation rates on naked DNA but very poorly on chromatin templates. J Biol Chem 1992; 267 : 13647-55.

79. Cojocaru M, Jeronimo C, Forget D, Bouchard A, Bergeron D, Côte P, Poirier GG, Greenblatt J, Coulombe B. Genomic location of the human RNA polymerase II general machinery: evidence for a role of TFIIF and Rpb7 at both early and late stages of transcription. Biochem I 2008; 409: 139-47.

80. Nudler E, Gottesman ME. Transcription termination and antitermination in E. coli. Genes Cells 2002; 7: 755-68.

81. Richard P, Manley JL. Transcription termination by nuclear RNA polymerases. Genes Dev 2009; 23: 1247-69.

82. Kuehner JN, Pearson EL, Moore C. Unravelling the means to an end: RNA polymerase II transcription termination. Nat Rev Mol Cell Biol 2011; 12: 283-94.

83. Russell J, Zomerdijk JC. RNA-polymerase-I-directed rDNA transcription, life and works. Trends Biochem Sci 2005; 30: 87-96.

84. Nielsen S, Yuzenkova Y, Zenkin N. Mechanism of eukaryotic RNA polymerase III transcription termination. Science 2013; 340: 1577-80.

85. Larson MH, Greenleaf WJ, Landick R, Block SM. Applied force reveals mechanistic and energetic details of transcription termination. Cell 2008; 132: 971-82.

86. Koslover DJ, Fazal FM, Mooney RA, Landick R, Block SM. Binding and translocation of termination factor rho studied at the singlemolecule level. J Mol Biol 2012; 423: 664-76.

87. Gaspard P. Template-directed copolymerization, random walks along disordered tracks, and fractals. Phys Rev Lett 2016; 117: 238101.

88. van Mameren J, Modesti M, Kanaar R, Wyman C, Wuite GJ, Peterman EJ. Dissecting elastic heterogeneity along DNA molecules coated partly with Rad51 using concurrent fluorescence microscopy and optical tweezers. Biophys J 2006; 91: L78-80.

89. van Mameren J, Gross P, Farge G, Hooijman P, Modesti M, Falkenberg $M$, Wuite GJ, Peterman EJ. Unraveling the structure of DNA during overstretching by using multicolor, single-molecule fluorescence imaging. Proc Natl Acad Sci USA 2009; 106: 18231-6.

90. Gross P, Laurens N, Oddershede LB, Bockelmann U, Peterman EJ, Wuite GJ. Quantifying how DNA stretches, melts and changes twist under tension. Nat Physics 2011; 7: 731-6.

91. La Porta A, Wang MD. Optical torque wrench: angular trapping, rotation, and torque detection of quartz microparticles. Phys Rev Lett 2004; 92: 190801.

92. Ma J, Bai L, Wang MD. Transcription under torsion. Science 2013; 340: 1580-3.

93. Sheinin MY, Li M, Soltani M, Luger K, Wang MD. Torque modulates nucleosome stability and facilitates $\mathrm{H} 2 \mathrm{~A} / \mathrm{H} 2 \mathrm{~B}$ dimer loss. Nat Commun 2013; 4: 2579. 